EPJ Web of Conferences 53, 11001 (2013)

DOI: $10.1051 /$ epjconf/20135311001

(C) Owned by the authors, published by EDP Sciences, 2013

\title{
Interdisciplinary science with large aperture detectors
}

\author{
Lawrence Wiencke ${ }^{1, \mathrm{a}}$ for the Pierre Auger Collaboration ${ }^{2, \mathrm{~b}}$ \\ ${ }^{1}$ Colorado School of Mines, Golden Colorado, USA \\ ${ }^{2}$ Observatorio Pierre Auger, Av. San Martín Norte 304, 5613 Malargüe, Argentina
}

\begin{abstract}
Large aperture detector systems to measure high energy cosmic rays also offer unique opportunities in other areas of science. Disciplines include geophysics such as seismic and volcanic activity, and atmospheric science ranging from clouds to lightning to aerosols to optical transients. This paper will discuss potential opportunities based on the ongoing experience of the Pierre Auger Observatory.
\end{abstract}

\section{INTRODUCTION}

The study of high energy extragalactic cosmic rays requires large aperture detectors because the flux of these particles is very low, especially for primary energies above $5 \times 10^{19} \mathrm{eV}$. This fact is reflected in both the construction of progressively larger instruments from Fly's Eye [1] to HiRes [2] to Telescope Array [3] to the Pierre Auger Observatory [4] (see also [5]), and in the interest in space-based instruments such as JEM-EUSO [6] and precursor missions TUS [7] and Tatiana [8, 9]. All of these efforts invoke a common measurement principle of using larger and larger areas of the troposphere as part of the detection system. Because these instruments observe the earth in unique ways as well as the cosmos, their scientific potential extends beyond astrophysics and particle physics.

\section{EXAMPLES FROM THE PIERRE AUGER OBSERVATORY}

Completed in 2008 after a five year construction period, the Pierre Auger Observatory is spread across an area of $3000 \mathrm{~km}^{2}$ near the base of the Argentinean Andes $\left(35.3^{\circ} \mathrm{S}\right.$ and $69.3^{\circ} \mathrm{W}, 1400 \mathrm{~m}$ asl). The shortest straight line distance between the center of the site and the Pacific ocean is $270 \mathrm{~km}$. The corresponding distance to the Atlantic ocean is $800 \mathrm{~km}$. Located $135 \mathrm{~km}$ to the south west is the active volcano Cerro Azul. In 1846 and 1932 it produced two of South America's largest eruptions. The mass ejected by the second exceeded that of the 1980 US Mt. St Helen's eruption by a factor of nine. The world's southernmost $5000 \mathrm{~m}$ mountain, El Sosneado, is visible at a distance of $75 \mathrm{~km}$ to the west. The site is also located within the South Atlantic Anomaly region [10].

\subsection{Interdisciplinary science with the surface detector}

The surface detector (SD) [11] features1660 instrumented tanks filled with ultra pure water and arranged in a triangular grid with $1.5 \mathrm{~km}$ spacing between nearest neighbors. Cherenkov light from charged particles can be detected by three 9" photomultiplier tubes (PMTs) that view the water from the top of the tank. An internal liner seals the tank and the PMTs against external light which allows the SD to

\footnotetext{
ae-mail: 1wiencke@mines.edu

bFor the full authorlist see Appendix "Collaborations" in this volume

This is an Open Access article distributed under the terms of the Creative Commons Attribution License 2.0, which permits unrestricted use, distribution, and reproduction in any medium, provided the original work is properly cited.
} 


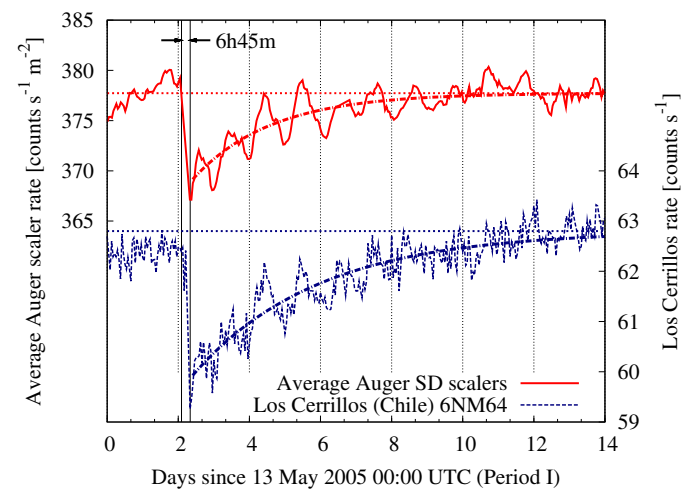

Figure 1. Example of a Forbush decrease that was observed by the Los Cirrilos neutron monitor station in Chile and as a decrease in the average scaler rate of the Pierre Auger Observatory surface detector.
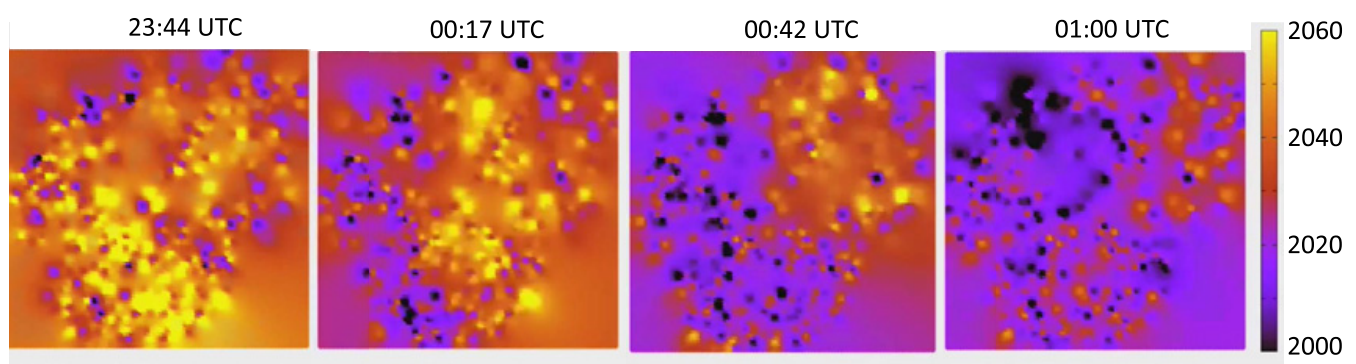

Figure 2. A storm on March 11-12 (UTC) 2009 crosses the Pierre Auger surface detector in this sequence of scaler rate maps. An animation is available [16].

operate with a duty cycle of $100 \%$. One percent of the high energy cosmic ray air shower events recorded by the SD are publicly available [12]. These data included showers with energies up to $5 \times 10^{19} \mathrm{eV}$ and zenith angles up to $60^{\circ}$. This on-line sample exceeds 25,000 events. An event viewer is also provided.

Most of the interdisciplinary science done to date with SD data has been derived from the measured singles rates, also called scaler rates. The singles rate is driven primarily by single muons from charged pions that were created by atmospheric interactions of abundant low energy cosmic rays $\left(\mathrm{E}=10^{9-12} \mathrm{eV}\right)$. The singles rate is about $3000 \mathrm{~Hz}$ per tank. The single triggers are not stored permanently, but their rates and the vertical equivalent muon (VEM) calibration factor are measured once per minute at each tank $[13,14]$. The scaler rate, averaged over all tanks in the SD and corrected for pressure effects, is publicly available in an ASCII file that is appended daily [15]. This file includes the scaler rate, pressure and temperature and other parameters averaged over 15 minute-intervals since March 30th 2005.

The SD scaler averages over the 1660 tanks represent more than 3 billion particles per interval. This sample size is so large that changes of order $0.1 \%$ are statistically significant. Changes on the order of $1-5 \%$ have been observed to correlate in time with a variety of physical phenomena. Decreases in the singles rate have been observed after massive solar flares that eject charged particles that alter the earth's magnetic field as shown in Fig. 1. The time correlation with measurements by neutron monitor stations is striking. Storms crossing the SD have also been observed to alter the singles rate (Fig. 2) as have high wind events. A study is also in progress to examine possible correlations between changes of the atmospheric electric field and changes in the SD scaler rates. 


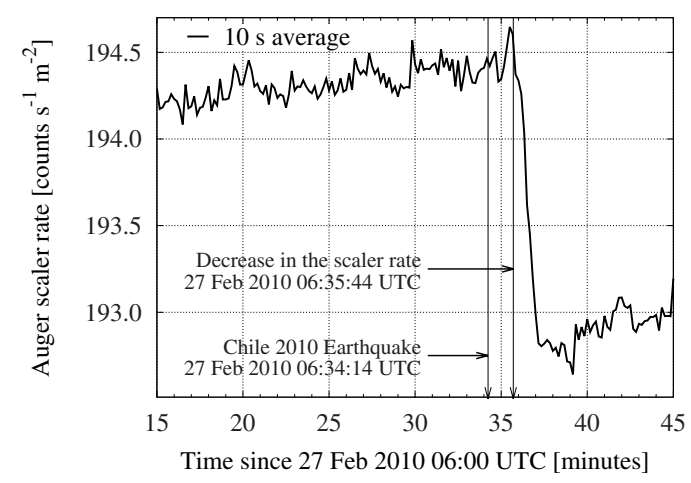

Figure 3. Following the Feb 27th 2010 earthquake off the coast of Chile, the scaler rate, averaged over all surface dectector tanks, dropped by $0.8 \%$. The statistical significance is estimated at more the $20 \sigma$ [18].

On Feb $27^{\text {th }} 2010$ a magnitude 8.8 earthquake occurred off the coast of central Chile [17]. After a delay comparable to the estimated transit time of the seismic waves from the epicenter to the observatory, the scaler rate dropped abruptly by $0.8 \%$ (Fig. 3) and recovered over several hours. Mechanisms that could have caused this decrease are being evaluated. The simplest explanation is that the scaler rate dropped, not because fewer charged particles entered the tanks, but because of some instrumental effect caused by the seismic shaking. The report of an apparent ionospheric electron enhancement preceding the 2011 March 11 Tohoku-Oki earthquake in Japan has been published [19]. This report provides additional motivation to better understand the coupling mechanism between the SD scaler rate and large earthquakes. In addition, during the 2012 Austral summer a temporary array of seismic detectors was deployed at the Observatory site and there are plans to install a permanent seismic array.

The SD and FD are sensitive to lightning. Since extensive air showers have been proposed as possible trigger mechanism a lightning detection system is planned. The data would be used to assist observatory operations and to search for correlations between lightning recorded by this system and extensive air showers recorded by the observatory.

\subsection{Interdisciplinary science with the fluorescence detector}

The fluorescence detector (FD) [20] overlooks the SD from four stations located along the Observatory boundary (Fig. 5 (Panel B)). The design is optimized to record the ultraviolet (UV) emission via nitrogen excitation from charged particles in cosmic ray extensive air showers. The field of view (FOV) and the viewing distance were optimized to achieve a large aperture. The six telescopes at each site view in total $180^{\circ}$ in azimuth by $30^{\circ}$ in elevation starting about $2^{\circ}$ above the horizon. At the Coihueco site three "HEAT" [21] telescopes extend the FOV to $60^{\circ}$ in elevation. Near the center of the Observatory where the CLF and XLF UV laser facilities [22, 23] are located, the 4-site FOV extends to about $17 \mathrm{~km}$ asl. Viewing distance was optimized in several ways. Optical clarity was an important criteria in the observatory site selection process. Each telescope has an optically filtered entrance aperture of $2.2 \mathrm{~m}$ diameter. The filter is optimized for 300-400 nm transmission to enhance the contrast of UV light emission of atmospheric nitrogen above the broader background spectrum of the night sky. A relatively coarse pixelization of $1.5^{\circ} \times 1.5^{\circ}$ per PMT and a $10 \mathrm{MHz}$ digitization rate optimizes performance per channel cost. Each telescope "camera" contains 440 PMTs. The PMTs are AC coupled. The dynamic range spans between 3 and $10^{5}$ photoelectrons per $100 \mathrm{~ns} .100 \mu \mathrm{s}$ (1000 bins) of digitized data for each pixel is stored in a ring buffer. The stored data drive a three tier trigger scheme to identify pulses above threshold and then identify track candidates that contain at least five adjacent triggered pixels. The triggering scheme reduces the data rate from a nominal $100 \mathrm{~Hz}$ singles rate per PMT to a trigger rate per 

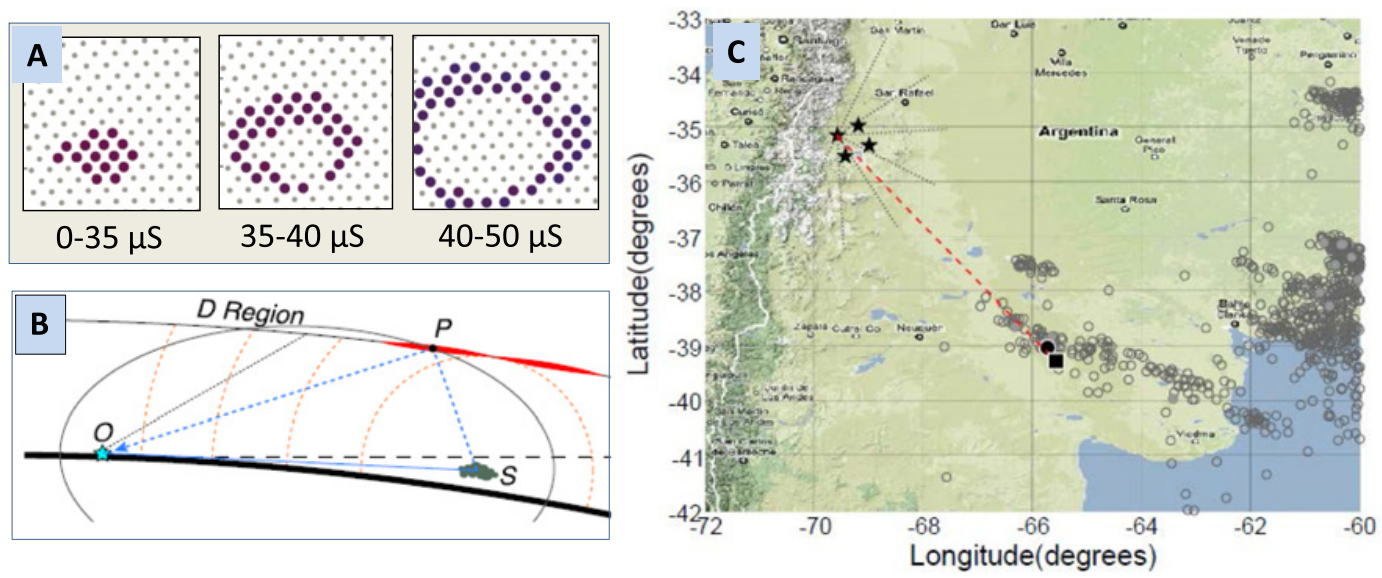

Figure 4. Observation and reconstruction of an atmospheric transient "Elf" by the fluorescence detector. The time evolution of the light image arriving at FD telescope PMT camera is shown in panel A. (A subsection of the camera is shown. The field of view of each PMT (black dots) is $1.5^{\circ} \times 1.5^{\circ}$ ). A geometrical model (panel B) used to reconstruct the position of the lightning (S) assumes an electromagnetic pulse travels from (S) to the emission point (P) and the emitted light travels to the observatory $(\mathrm{O})$. The lightning strike is below the horizon (dashed line).The reconstructed position of the elf (panel C) is well outside the observatory. (The four stars represent the four FD sites.) The black square is the reconstructed position of (S). Circles represent the positions of lightning strikes on the same day recorded by the World Wide Lightning Location Network. The black circle represents the position of lightning that occurred on the same second that the FD observed the elf.
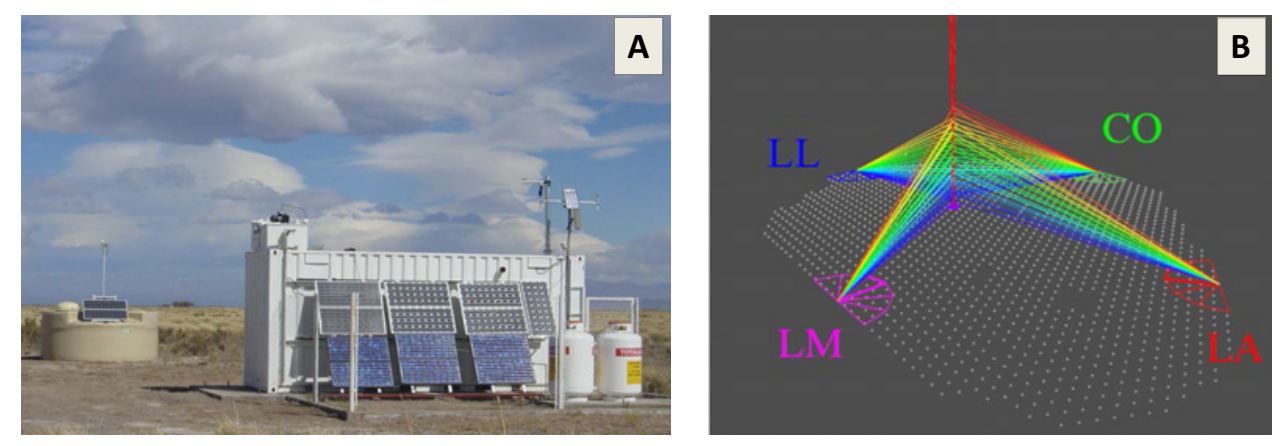

Figure 5. The Central Laser Facility (panel A) directs calibrated pulses of $355 \mathrm{~nm}$ light into the atmosphere. For nearly vertical directions, the light scattered out of the beam by the atmosphere can be observed by telescopes at all four FD sites (panel B) in a four-fold bi-static LIDAR configuration. (LL, CO, LA, and LM denote the four FD sites.)

FD site of about $0.02 \mathrm{~Hz}$, not including the laser tracks recorded from the CLF (Fig. 5 (Panel A)) and XLF on a typical night.

Extensive air showers are not the only type of optical transient that the FD has measured. Transient luminous events called "Elves" [24] have also been observed [25]. These are believed to be optical emission high above some thunderstorms from an electron excitation process in the D layer of the ionosphere. An electromagnetic pulse from the thunderstorm causes the excitation. Although just a few have been recorded to date by the FD, an improvement to the FD trigger algorithm is expected to increase the number significantly. An example shown in Fig. 4 was reconstructed from the time evolution of the FD optical signature. The result placed the lightning more than $500 \mathrm{~km}$ away, but near a lightning event 

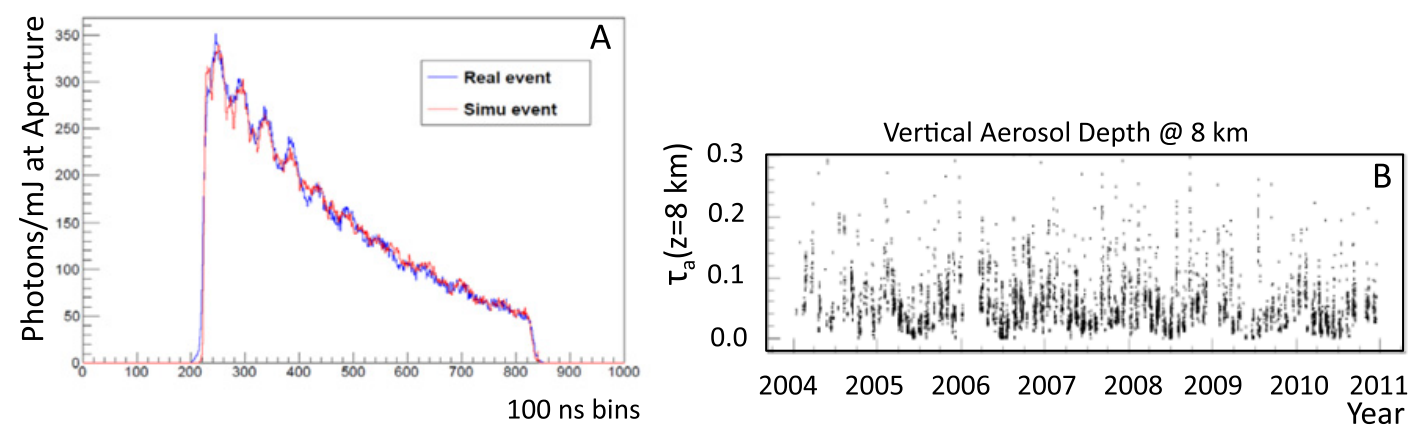

Figure 6. The data shown in panel A were collected during very clear conditions by the Pierre Auger Observatory fluorescence detector. It shows the level of agreement between the signal recorded by an FD telescope averaged over 50 vertical CLF laser pulses (Fig. 5 (Panel B)) and the simulated signal assuming an aerosol-free atmosphere. The aerosol optical depth as a function of height can be determined by comparing the observed profile with a reference profile from a real or simulated clear period. Seasonal variations are evident in the seven year record of sample data from the hourly aerosol database (panel B). This example shows measurements from a telescope at the Los Leones FD site.
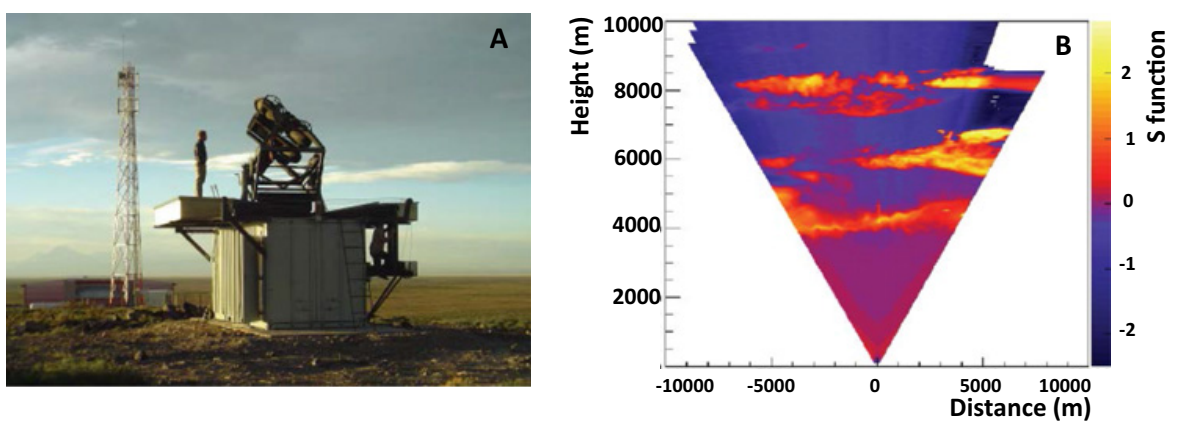

Figure 7. One of the four steerable elastic backscatter LIDAR systems is shown in panel A. The Los Morados FD installation is visible in the background. A set of LIDAR scans from one system were analyzed with a cloud edge detection algorithm to obtain the false color images of cloud layers shown in panel B.

recorded independently by the World Wide Local Lightning Location Network [26] on the same second as the FD observation. The $100 \mathrm{~ns}$ time resolution of the FD, large optical aperture, and full horizon coverage that is essentially doubled beyond the SD footprint offer significant potential to advance the understanding of such transient luminous events.

The Observatory is supported by a program of atmospheric monitoring that is arguably the most extensive located in the southern hemisphere. The two laser stations, four back-scatter LIDARs [27], radiosonde launches, IR cameras, weather stations and other instruments, including the FD itself, are used to characterize bulk properties of the essential atmospheric components: molecular [28, 29], suspended aerosols [30], and clouds. (See also [31].) Ongoing research of an interdisciplinary nature includes validation of atmospheric extrapolations models using Global Data Assimilation System (GDAS) [32], development of methods to measure aerosol optical depth under very clear conditions and the accumulation of more than seven years of aerosol optical depth profile measurements using a novel bi-static LIDAR technique (Fig. 6), development of methods to scan for clouds (Fig. 7) and aerosols shortly after the detection of selected cosmic ray events, using FD measurements of cloud illumination by the CLF to provide ground truth comparison with GOES satellite data. An upgrade in progress [33] 
will add a Raman LIDAR receiver to the CLF to compare the techniques developed at the observatory with the best practices method used in the atmospheric sciences community to measure aerosol optical depth. In addition, a proposed project under consideration would combine aerosol measurements from the Auger Observatory with local aerosol sampling measurements to study the origin and transport of iron-bearing aerosols that play a role in biological processes in the southern ocean.

\section{OUTLOOK}

The interdisciplinary program of the Pierre Auger Observatory is expected to grow over the next years as more data becomes public and new instruments come on-line at the site. The collaboration welcomes interested partners in these efforts, and is of course interested in new ideas. Proceedings from the 2011 workshop on Interdisciplinary Science at the Auger Observatory (IS@AO) [34] are expected to be available this year (2012).

The author thanks the UHECR 2012 organizers for their hospitality, and the Pierre Auger Observatory staff for their invaluable partnership. Part of this work is supported through NSF grant 0855680.

\section{References}

[1] R.M. Baltrusaitis et al., Nucl. Instr. Meth. A 20 (1985) 410

[2] J. Boyer et al. Nucl. Instr. Meth. A 482 (2002) 410

[3] H. Kawai et al., Nucl. Phys. Proc. Suppl. 175-176 (2008) 253

[4] The Pierre Auger Collaboration, Nucl. Instr. Meth. A 523 (2004) 50

[5] K. Nakamura et al. (Particle Data Group), Phys. G 37 (2010) 075021 (http://pdg.lbl.gov/ 2011/reviews/rpp2011-rev-particle-detectors-non-accel.pdf)

[6] T. Ebisuzaki for the JEM-EUSO Collaboration, Proc. 32nd ICRC 3 (2011) 10 [arXiv:1204.5065v1 [astro-ph.IM]]

[7] L. Tkachev et al. Proc. 32nd ICRC V 3 (2011) 121

[8] G. K. Garipov et al., J. Geophys. Res. 115 (2010) A00E24 [doi:10.1029/2009JA014765] 45 (2007) 273

[9] G. K. Garipov et al. Cosmic Research 45 (2011) 391

[10] F. Fürst, et al., Earth \& Planet. Sci. Lett., 281 (3-4) (2009) 125 [arXiv:0902.2873v1 [astro-ph.EP]]

[11] The Pierre Auger Collaboration, Nucl. Instr. Meth. A 586 (2008) 409

[12] http://auger.colostate.edu/ED/

[13] X. Bertou, et al., for the Pierre Auger Collaboration, Nucl. Instr. Meth. A 568 (2006) 83

[14] The Pierre Auger Collaboration, JINST 6 (2011) 01003

[15] http://auger.colostate.edu/ED/scaler.php

[16] http://bit.ly/augerstorm

[17] C. Vigny et al., Science 332 (2011) 1417

[18] H. Asorey for the Pierre Auger Collaboration, Proc. 32nd ICRC 11 (2011) 462 [arXiv:1107.4805v1 [astro-ph.HE]]

[19] K. Heki, Geophys. Res. Lett., 38 (2011) L17312 [doi:10.1029/2011GL047908]

[20] The Pierre Auger Collaboration, Nucl. Instr. Meth. A 620 (2010) 227 [arXiv:0907.4282v1

[21] C. Meurer, N. Scharf on behalf of the Pierre Auger Collaboration, Astrophys. Space Sci. Trans. 7 (2011) 183 [arXiv:1106.1329v1 [astro-ph.IM]]

[22] B. Fick et al. JINST 1 (2006) P11003 [arXiv:astro-ph/0507334v1]

[23] http://astroserve.mines.edu

[24] A. Chen et al. J. Geophy. Res. 113 (208) A08306 [doi:10.1029/2008JA013101] 


\section{UHECR 2012}

[25] A. Tonachini for the Pierre Auger Collaboration, Proc. 32nd ICRC 11 (2011) 400 [arXiv:1107.4806v1 [astro-ph.IM]]

[26] http://webflash.ess.washington.edu/

[27] S. Benzvi et al. Nucl. Instr. Meth A 574 (2007) 171 [arXiv:astro-ph/0609063v2]

[28] The Pierre Auger Collaboration, Astropart. Phys. 32 (2009) 89 [arXiv:0906.5497v2 [astroph.IM]]

[29] The Pierre Auger Collaboration, Astropart. Phys. 35 (2012) 591 [arXiv:1201.2276 [astro-ph.HE]]

[30] The Pierre Auger Collaboration, Astropart. Phys. 33 (2010) 108 [arXiv:1002.0366 [astro-ph.IM]]

[31] The Pierre Auger Collaboration, Proc. 32nd ICRC (2011) [arXiv:1107.4806v1 [astro-ph.IM]]

[32] NOAA Air Resources Laboratory (ARL), Global Data; Assimilation System (GDAS) Archive Information, Tech. Rep. (2004); http://ready.arl.noaa.gov/gdas1.php

[33] L. Wiencke for the Pierre Auger Collaboration, Proc 32nd ICRC 3 (2011) 141 [arXiv:1107.4806v1 [astro-ph.IM]]

[34] http://www.auger.org/ISatAO/ 